\title{
Two types of excited electron dynamics in zinc oxide
}

\author{
V. P. Zhukov, ${ }^{1,2}$ P. M. Echenique, ${ }^{2,3,4}$ and E. V. Chulkov²,3,4 \\ ${ }^{1}$ Institute of Solid State Chemistry, Urals Branch of the Russian Academy of Sciences, \\ Pervomayskaya 91, GSP-145, Yekaterinburg, Russia \\ ${ }^{2}$ Departamento de Física de Materiales, Facultad de Ciencias Químicas, UPV/EHU, \\ Apdo. 1072, San Sebastián, 20080 Basque Country, Spain \\ ${ }^{3}$ Centro de Fisica de Materiales (CFM-MPC) and Centro Mixto CSIC, UPV/EHU, \\ Apdo. 1072, San Sebastián, 20080 Basque Country, Spain \\ ${ }^{4}$ Donostia International Physics Center (DIPC), P. Manuel de Lardizabal, 4, San Sebastián, 20018 Basque Country, Spain
}

(Received 7 June 2010; revised manuscript received 13 July 2010; published 2 September 2010)

\begin{abstract}
We present first-principle evaluations of the electron-phonon coupling strength parameter and associated characteristics of relaxation for the excited electrons in the conduction band of zinc oxide. The evaluations are based on the pseudopotential plane-wave approach to the electronic band structure, the density-functional perturbation theory for the calculations of phonons and electron-phonon interactions, and on the "Fermi golden rule" for evaluations of the electron relaxation time and the energy-loss time. The calculations demonstrate existence of two types of electron dynamics, the picosecond one for electrons near the bottom of the conduction band and the femtosecond for the higher energies. Sensibly good agreement with experimental data confirms the validity of the calculations.
\end{abstract}

DOI: 10.1103/PhysRevB.82.094302

PACS number(s): 77.55.hf, 71.38.-k, 78.47.D-, 78.47.J-

\section{INTRODUCTION}

A great interest of researchers in zinc oxide is supported by the perspectives of its applications in optical and optoelectronic devices. ${ }^{1,2}$ Besides, the doped zinc oxide manifests itself as a good photocatalyst which can be used for decomposing harmful bacteria and organic contaminants. ${ }^{3-5}$ Since the optical and photocatalytic properties to a great extent depend on the dynamics of excited states, the studies of fast electron dynamics in zinc oxide have been carried out in a series of experimental works employing time-resolved optical techniques. A great part of them concerns with the dynamics of exciton states important for optical applications. ${ }^{6-11}$ These researches have permitted to evaluate the rates of the main excitonic processes, i.e., relaxation, recombination, and capture by impurities. Since the concentration of excitons in oxide semiconductors is typically low, in the photocatalytic processes more important is the dynamics of free excited carriers, electrons and holes. However, the available experimental data are controversial. Yamamoto et $a l .{ }^{12}$ performed two-photon time-resolved experiments with the energy of quanta from 3 to $3.5 \mathrm{eV}$ and deduced the time of cooling of the electron-hole plasma in $\mathrm{ZnO}$ shorter than 1 ps. Sun et al. ${ }^{13}$ studied the carrier dynamics in $\mathrm{ZnO}$ in the excitation energy interval from 3.33 to $3.48 \mathrm{eV}$ and found at $3.33 \mathrm{eV}$ a faster thermalization time, about $200 \mathrm{fs}$. In the pump-probe experiments of Wen et al. ${ }^{6}$ the electron relaxation time at room temperature has been estimated as about $800 \mathrm{fs}$ at the energy above $3.45 \mathrm{eV}$ but essentially increasing with the reduction in energy, up to $34 \mathrm{ps}$ at $3.26 \mathrm{eV}$. Hendry et al. ${ }^{9}$ performed for $\mathrm{ZnO}$ the pump-probe experiments with probing in tetrahertz energy region. At temperature about 20 $\mathrm{K}$ and excitation energy of $3.21 \mathrm{eV}$, i.e., below the fundamental band gap, they found the time of binding of free electrons and holes into excitons as 20 ps.

With such great dispersion of experimental estimates, it is desirable to perform first-principle theoretical calculations of the characteristic times of carrier dynamics in $\mathrm{ZnO}$. Since the value of the energy gap in wurtzite-structure $\mathrm{ZnO}$ is $3.37 \mathrm{eV}$, the main mechanism of electron relaxation at the energy of excited electrons with respect to the bottom of the conduction band (BCB) less than $3.37 \mathrm{eV}$ is the electron-phonon interaction. This is the main distinction of the electron relaxation in semiconductors from the relaxation in metals, where the main mechanism of relaxation is the electron-electron scattering well studied before on the first-principle level ${ }^{14-16}$ One can evaluate the electron relaxation time in semiconductors using the theory of electron-phonon interactions in metals. ${ }^{17,18}$ The chance to perform such calculations has appeared with the development of the first-principle methods of calculating the phonon spectra and electron-phonon scattering based on the density-functional perturbation theory. ${ }^{19,20}$ However, we are aware of only two evaluations of this type: for excited electrons in GaAs, GaSb, and GaP (Refs. 21 and 22) and for electrons in the conduction bands of the e-doped $\mathrm{LiH} .{ }^{23}$ In contrast to Ref. 22, our approach includes the evaluations of the momentum-averaged electron-phonon relaxation rate in $\mathrm{ZnO}$ in two ranges of excited electron energy. The first range extends from the $\mathrm{BCB}$ to the maximum phonon energy and the second range extends from the top energy of the first range to the energy $3.0 \mathrm{eV}$ with respect to the $\mathrm{BCB}$, i.e., up to the energy close to that at which the electron-electron scattering becomes essential. We find that the rate of electron-phonon relaxation significantly differs in these two energy regions: whereas in the first region it happens in the picosecond time scale, in the second one it occurs in the femtosecond time scale.

\section{THEORY}

In accordance to the "golden Fermi rule," the probability, per unit of time, of the electron transition between two states 
mediated by the electron-phonon coupling is determined by the equation

$$
P_{\mathbf{k}, i ; \mathbf{k}+\mathbf{q}, j}^{\nu}=(2 \pi / \hbar)\left|\left\langle\mathbf{k}, i\left|\Delta V_{\mathbf{q} \nu}\right| \mathbf{k}+\mathbf{q}, j\right\rangle\right|^{2} \delta\left(e_{\mathbf{k}+\mathbf{q}, j}-e_{\mathbf{k}, i}\right) .
$$

Here $|\mathbf{k}, i\rangle$ and $|\mathbf{k}+\mathbf{q}, j\rangle$ are the electronic states participating in the transition accompanied by the absorption or emission of phonon with the moment $\mathbf{q}$ and polarization $\nu$, and the value $\Delta V_{\mathbf{q} \nu}$ is the variation in the self-consistent potential in crystal caused by the displacement mode of the phonon. The value $\left\langle\mathbf{k}, i\left|\Delta V_{\mathbf{q} \nu}\right| \mathbf{k}+\mathbf{q}, j\right\rangle$ is the matrix element of the electron-phonon interaction; the ways of calculating this matrix element have been extensively discussed in literature. ${ }^{20}$ In Eq. (1) the energy of the phonon has been neglected since it is small compared to the energies of electronic states $e_{\mathbf{k}+\mathbf{q}, j}$, $e_{\mathbf{k}, j}$; this is the so-called quasielastic approximation. Having integrated over all the possible momenta of phonons with energy $\omega$, one obtains the probability for the electron to leave the state $|\mathbf{k}, i\rangle$ (probability normalization integral is temporarily omitted; it is discussed later)

$$
\begin{aligned}
\Phi_{\mathbf{k}, i}(\omega)= & (2 \pi / \hbar) \sum_{j} \sum_{\nu} \int_{B Z} \delta\left(\omega_{\mathbf{q}}^{\nu}-\omega\right) \mid\left\langle\mathbf{k}, i\left|\Delta V_{\mathbf{q} \nu}\right| \mathbf{k}\right. \\
& +\mathbf{q}, j\rangle\left.\right|^{2} \delta\left(e_{\mathbf{k}+\mathbf{q}, j}-e_{\mathbf{k}, i}\right) d^{3} \mathbf{q} .
\end{aligned}
$$

Here and in all the following equations the integration over $\omega$ extends from zero to the maximum phonon energy. Integrating over $\omega$ and taking into account thermodynamic factors for electrons and phonons one obtains the expression for the rate of electron-phonon relaxation

$$
\begin{aligned}
\Gamma_{\mathbf{k}, i}= & \int d \omega \Phi_{\mathbf{k}, i}(\omega)\left[1+2 n(\omega, T)+f\left(\omega+e_{\mathbf{k}, i}, T\right)-f(\omega\right. \\
& \left.\left.-e_{\mathbf{k}, i}, T\right)\right] .
\end{aligned}
$$

In Eq. (3) the probability of phonon emission and absorption has been taken into account; it depends on temperature through the Bose-Einstein function for phonons $n(\omega, T)$ and the Fermi-Dirac function for electrons $f(\omega, T)$, see details in. ${ }^{17}$ At low temperature it is reduced, with a high precision, to the equation

$$
\begin{aligned}
\Gamma_{\mathbf{k}, i}= & \int d \omega \Phi_{\mathbf{k}, i}(\omega)[2 n(\omega, T)+1] \\
= & (2 \pi / \hbar) \sum_{j} \sum_{\nu} \int_{B Z} d \omega \int_{B Z} d^{3} \mathbf{q} \delta\left(\omega_{\mathbf{q} \nu}-\omega\right) \mid\left\langle\mathbf{k}, i\left|\Delta V_{\mathbf{q} \nu}\right| \mathbf{k}\right. \\
& +\mathbf{q}, j\rangle\left.\right|^{2} \delta\left(e_{\mathbf{k}+\mathbf{q}, j}-e_{\mathbf{k}, i}\right)[2 n(\omega, T)+1] \\
= & (2 \pi / \hbar) \sum_{j} \sum_{\nu} \int_{B Z} d^{3} \mathbf{q} \delta\left(e_{\mathbf{k}+\mathbf{q}, j}-e_{\mathbf{k}, i}\right) \mid\left\langle\mathbf{k}, i\left|\Delta V_{\mathbf{q} \nu}\right| \mathbf{k}\right. \\
& +\mathbf{q}, j\rangle\left.\right|^{2}\left[2 n\left(\omega_{\mathbf{q} \nu}, T\right)+1\right] .
\end{aligned}
$$

The function $\Phi_{\mathbf{k}, i}(\omega)$ differs only in a constant from the wellknown Eliashberg function $\alpha^{2} F_{\mathbf{k}, i}(\omega)=\Phi_{\mathbf{k}, i}(\omega) /(2 \pi / \hbar)$,

$$
\begin{aligned}
\alpha^{2} F_{\mathbf{k}, i}(\omega)= & \sum_{j} \sum_{\nu} \int_{B Z} \delta\left(\omega_{\mathbf{q}}^{\nu}-\omega\right)\left|\left\langle\mathbf{k}, i\left|\Delta V_{\mathbf{q} \nu}\right| \mathbf{k}+\mathbf{q}, j\right\rangle\right|^{2} \delta\left(e_{\mathbf{k}+\mathbf{q}, j}\right. \\
& \left.-e_{\mathbf{k}, i}\right) d^{3} \mathbf{q} .
\end{aligned}
$$

The Eliashberg function determines the electron-phonon interaction strength parameter

$$
\lambda_{\mathbf{k}, i}=2 \int \frac{\alpha^{2} F_{\mathbf{k}, i}(\omega)}{\omega} d \omega .
$$

The time of electronic relaxation for the state $|\mathbf{k}, i\rangle$ is determined as the inverse value of the rate of electronphonon relaxation

$$
\tau_{\mathbf{k}, i}=\hbar / \Gamma_{\mathbf{k}, i} .
$$

So $\tau_{\mathbf{k}, i}$ characterizes the time that the electron can reside in the state $|\mathbf{k}, i\rangle$. The values $\Gamma_{\mathbf{k}, i}$ and $\tau_{\mathbf{k}, i}$ characterize the quasielastic scattering of an electron via phonon emission and absorption. The $\Gamma_{\mathbf{k}, i}$ value also determines the width of the electronic spectral line. ${ }^{17}$

In the case of photocatalyses we are also interested in evaluating the energy-loss time, hereafter $\tau_{e n}$, which is the time necessary for an electron to fall down to the bottom of the conduction band via repeated emission of phonons. One can evaluate the averaged loss of energy by the electron in the state $|\mathbf{k}, i\rangle$; it is determined by the first moment of the probability function $\Phi_{\mathbf{k}, i}$

$$
\begin{aligned}
\Delta e_{\mathbf{k}, i}= & \int d \omega \Phi_{\mathbf{k}, i}(\omega) \omega[2 n(\omega, T)+1] \\
= & 2 \pi \int d \omega \alpha^{2} F_{\mathbf{k}, i}(\omega) \omega[2 n(\omega, T)+1] \\
= & 2 \pi \int d \omega \omega \sum_{j} \sum_{\nu} \int_{B Z} d^{3} \mathbf{q} \delta\left(\omega_{\mathbf{q}}^{\nu}-\omega\right) \mid\left\langle\mathbf{k}, i\left|\Delta V_{\mathbf{q} \nu}\right| \mathbf{k}\right. \\
& +\mathbf{q}, j\rangle\left.\right|^{2} \delta\left(e_{\mathbf{k}+\mathbf{q}, j}-e_{\mathbf{k}, i}\right)\left[2 n\left(\omega_{\mathbf{q} \nu}, T\right)+1\right] \\
= & 2 \pi \sum_{j} \sum_{\nu} \int_{B Z} d^{3} \mathbf{q} \omega_{\mathbf{q} \nu}\left|\left\langle\mathbf{k}, i\left|\Delta V_{\mathbf{q} \nu}\right| \mathbf{k}+\mathbf{q}, j\right\rangle\right|^{2} \delta\left(e_{\mathbf{k}+\mathbf{q}, j}\right. \\
& \left.-e_{\mathbf{k}, i}\right)\left[2 n\left(\omega_{\mathbf{q} \nu}, T\right)+1\right] .
\end{aligned}
$$

The integration over $\mathbf{q}$ includes the whole Brillouin zone. (It is accepted hereafter that the atomic system of units is employed, so $\hbar=1$ and energy is in Ry units.)

One can obtain then the expression for the momentumaveraged values. For the electron-phonon coupling parameter it is

$$
\lambda(\epsilon)=[1 / N(\epsilon)] \sum_{i} \int d^{3} \mathbf{k} \delta\left(e_{\mathbf{k}, i}-\epsilon\right) \lambda_{\mathbf{k}, i} \Omega_{\mathbf{k}, i} .
$$

By analogy, for relaxation rate we have

$$
\Gamma(\epsilon)=[1 / N(\epsilon)] \sum_{i} \int d^{3} \mathbf{k} \delta\left(e_{\mathbf{k}, i}-\epsilon\right) \Gamma_{\mathbf{k}, i} / \Omega_{\mathbf{k}, i}
$$

and for the energy loss we have 


$$
\Delta e(\epsilon)=[1 / N(\epsilon)] \sum_{i} \int d^{3} \mathbf{k} \delta\left(e_{\mathbf{k}, i}-\epsilon\right) \Delta e_{\mathbf{k}, i} / \Omega_{\mathbf{k}, i} .
$$

Here $N(\epsilon)$ is the density of electronic states at the energy $\epsilon$, and we have taken into account the probability normalization integral $\Omega_{\mathbf{k}, i}$

$$
\Omega_{\mathbf{k}, i}=\int d \omega \Phi_{\mathbf{k}, i}(\omega)
$$

Then one obtains

$$
\begin{aligned}
\Gamma(\epsilon)= & {[2 \pi / N(\epsilon)] \sum_{i} \sum_{j} \sum_{\nu} \int_{B Z} d^{3} \mathbf{k} \int d^{3} \mathbf{q} \delta\left(e_{\mathbf{k}, i}-\epsilon\right) \delta\left(e_{\mathbf{k}+\mathbf{q}, j}\right.} \\
& -\epsilon)\left|\left\langle\mathbf{k}, i\left|\Delta V_{\mathbf{q} \nu}\right| \mathbf{k}+\mathbf{q}, j\right\rangle\right|^{2}\left[2 n\left(\omega_{\mathbf{q} \nu}, T\right)+1\right] / \Omega_{\mathbf{k}, i}, \\
\Delta e(\epsilon)= & {[2 \pi / N(\epsilon)] \sum_{i} \sum_{j} \sum_{\nu} \int_{B Z} d^{3} \mathbf{k} \int d^{3} \mathbf{q} \delta\left(e_{\mathbf{k}, i}-\epsilon\right) \delta\left(e_{\mathbf{k}+\mathbf{q}, j}\right.} \\
& -\epsilon)\left|\left\langle\mathbf{k}, i\left|\Delta V_{\mathbf{q} \nu}\right| \mathbf{k}+\mathbf{q}, j\right\rangle\right|^{2} \omega_{\mathbf{q} \nu}\left[2 n\left(\omega_{\mathbf{q} \nu}, T\right)+1\right] / \Omega_{\mathbf{k}, i} .
\end{aligned}
$$

In order to calculate the normalization integral $\Omega_{\mathbf{k}, i}$ we employ the averaging over all the energies $\epsilon_{\mathrm{k}, i}=\epsilon$, i.e., we admit that this integral can be replaced with

$$
\Omega(\epsilon)=[1 / N(\epsilon)] \sum_{i} \int d^{3} \mathbf{k} \delta\left(e_{\mathbf{k}, i}-\epsilon\right) \Omega_{\mathbf{k}, i} .
$$

This is the approximation similar to the "random $k$-vector" approximation, successful in the theory of femtosecond electron dynamics in metals. ${ }^{24}$ It simplifies and accelerates the calculations. Eventually the expression for $\Delta e(\epsilon)$ is reduced to

$$
\Delta e(\epsilon)=\frac{\sum_{\nu} \int d^{3} \mathbf{q} \omega_{\mathbf{q}, \nu} \widetilde{\Gamma}_{\mathbf{q}, \nu}(\epsilon)}{\sum_{\nu} \int d^{3} \mathbf{q} \widetilde{\Gamma}_{\mathbf{q}, \nu}(\epsilon)},
$$

where

$$
\begin{aligned}
\widetilde{\Gamma}_{\mathbf{q}, \nu}(\boldsymbol{\epsilon})= & {[2 \pi / N(\epsilon)] \sum_{i} \sum_{j} \sum_{\nu} \int_{B Z} d^{3} \mathbf{k} \delta\left(e_{\mathbf{k}, i}-\epsilon\right) \delta\left(e_{\mathbf{k}+\mathbf{q}, j}-\epsilon\right) } \\
& \times\left|\left\langle\mathbf{k}, i\left|\Delta V_{\mathbf{q} \nu}\right| \mathbf{k}+\mathbf{q}, j\right\rangle\right|^{2} / \Omega(\epsilon)
\end{aligned}
$$

is the $\mathbf{q}, \nu$ th term of the integral [Eq. (13)] with omitted temperature-dependent factor $2 n+1$. One can evaluate then the time $\tau_{\text {en }}$ necessary for an electron to fall down to the bottom of the conduction band. If the time of electron relaxation is $\tau(\epsilon)$, and the effective energy loss is $\Delta e(\epsilon)$, then the rate of energy loss is $\Delta e(\epsilon) / \tau(\epsilon)=\Delta e(\epsilon) \Gamma(\epsilon)$. Then the time of loosing the total excess energy (which we define as the energy of the excited electron with respect to $\mathrm{BCB}$ ) is

$$
\tau_{e n}(\epsilon)=\int_{0}^{\epsilon} \frac{d E}{\Delta e(E) \Gamma(E)} .
$$

The foregoing theory is based on the assumption that the excess energy of an excited electron is higher than the maximum phonon energy. In this case any optical or acoustic phonon can be excited in the course of electron relaxation, so the integration over phonon momenta extends over the whole Brillouin zone. This is not valid if the excess energy of the electron is less that the maximum phonon energy. In such the case the phase space of phonons which can be excited is reduced by the requirement of the energy conservation. For this energy region we disregard the differences in the values of the electron-phonon matrix elements and evaluate the electron relaxation rates from only the value of the phonon phase space. The low-energy relaxation rate is then evaluated as

$$
\Gamma(\epsilon)=N_{p h}(\epsilon) \Gamma\left(\omega_{\max }\right) .
$$

Here $\Gamma\left(\omega_{\max }\right)$ is the relaxation rate of an electron whose excess energy is equal to the maximum phonon energy $\omega_{\max }$, and $N_{p h}(\epsilon)$ is the total number of phonon states (i.e., the phase space) in the energy interval from zero to $\epsilon$, normalized to unity at $\epsilon=\omega_{\max }$. We calculate the value $\Gamma\left(\omega_{\max }\right)$ as it was discussed before for the high-energy region. Afterwards we evaluate the energy-loss time following Eq. (7).

\section{DETAILS OF CALCULATIONS}

The numerical evaluations have been done using the density-functional perturbation theory ${ }^{19,20}$ implemented in the pseudopotential QUANTUM ESPRESSO (QE) computer $\operatorname{code}^{25}$ where we have included all the modifications necessary for our purposes. In the calculations of electronic states the ultrasoft pseudopotentials with gradient-corrected exchange-correlation of Perdue and Wang provided by the $\mathrm{QE}$ for the zinc and oxygen atoms were employed, hence, $\mathrm{Zn} 3 d$ states were treated as valence ones. A plane-wave basis set with the energy cutoff of 50 Ry was employed in the electronic-structure calculations, which is sufficient for the calculations of phonon frequencies, as it was shown in Refs. 26 and 27. Additionally, we found that the electronic band structure does not practically change if the cutoff energy is increased up to $80 \mathrm{Ry}$. The calculations were performed for the optimized ground state of the wurtzite structure. The total optimization of the crystal structure parameters has resulted in very small deviations from the experimental data, less than $0.5 \%$. It is not clear a priory how dense should be the wave vector grid for electrons $(\mathbf{k})$ and phonons $(\mathbf{q})$ to provide a good convergence of the electron-phonon matrix elements and all the dependent values. So we have checked the electron and phonon band structure calculations with three different Monkhorst-Pack grids. The grids $6 \times 6 \times 4,8 \times 8$ $\times 6$, and $10 \times 10 \times 8$ of $\mathbf{q}$ vectors and $\mathbf{k}$ vectors have been employed for the phonon calculations while the grids of $\mathbf{k}$ vectors denser by a factor of 2 have been employed in the calculations of electron-phonon matrix elements. It is shown in the following section that a very small difference in the characteristics of electron dynamics is achieved when going from the second to the third grid, which is denser than those employed in previous works on the phonon calculations for the oxide semiconductors. ${ }^{26,27}$ So we believe that with the 
third grid the momentum-averaged characteristics of electron dynamics are practically converged. With all the three grids we have the electronic band structure similar to that obtained in previous density-functional theory-local-density approximation (DFT-LDA) calculations. ${ }^{28}$ The main drawback of such calculations is a too small value of the electronic band gap, $1.2 \mathrm{eV}$ in our calculations as compared with the experimental value of $3.4 \mathrm{eV}$. Much better value of the band gap can be obtained by applying the so-called many-body GWcorrections to the DFT-LDA band structure. ${ }^{29}$ We do not believe, however, that the characteristics of electron dynamics in $\mathrm{ZnO}$ can be changed with the GW corrections. The variation in the self-consistent potential $\Delta V_{\mathbf{q} \nu}$ defining the probability of electronic transitions via the Eq. (1) is determined by the occupied states, so it is independent on the value of the band gap. On the other hand, within the GW approach the electronic state wave functions are kept equal to those obtained in the DFT-LDA band-structure methods. Hence one may expect that the momentum-averaged electronic transition probabilities between the conduction band states can be changed with inclusion of the GW corrections merely if the dispersion of these states is essentially changed. This is, however, not the case of $\mathrm{ZnO}$, where the GW corrections are small for the states in the valence band whereas the states of the conduction band are shifted with these corrections almost rigidly to higher energy. ${ }^{28,30}$

The delta function in Eqs. (13), (14), and (17) was approximated by the first-order Hermite-Gaussian function with a broadening width $\eta$. The choice of the broadening width $\eta$ provides a delicate problem of the calculations. This choice has to provide a good quality for momentum averaging of the electron-phonon interaction characteristics. A proper choice has been done using the results of the calculations performed for three energies of excited electron. These energies were $0.03,1.53$, and $3.03 \mathrm{eV}$ above the $\mathrm{BCB}$, hence close to the minimum, middle and maximum of the energy interval of interest. The choice of $\eta$ is discussed in the next section. All the calculations of the relaxation rate have been performed for zero temperature, that is with the $n(\omega, T=0)$ $=0$. Hence, all the effects associated with the thermal dilations of the crystal lattice, e.g., the temperature dependence of the electron-phonon relaxation rate, ${ }^{31}$ have been omitted.

\section{RESULTS AND DISCUSSIONS}

Since the first-principle calculations of the electronphonon interactions in semiconductors available in literature are scarce, a special attention in our research has been paid to the convergence of the momentum-averaged indices of electron dynamics with respect to the calculation parameters, first of all with respect to the number of phonon wave vectors and broadening parameter $\eta$. In Fig. 1 the density of phonon states (DOS) is shown for the three wave vector grids, $6 \times 6 \times 4,8 \times 8 \times 6$, and $10 \times 10 \times 8$. It is evident that the calculations are on the average well converged with respect to the number of $\mathbf{q}$ vectors. In Fig. 2 we show the computed phonon dispersion along the symmetry directions of the Brillouin zone together with the available experimental phonon frequencies. ${ }^{32-34}$ The mean-square deviation of

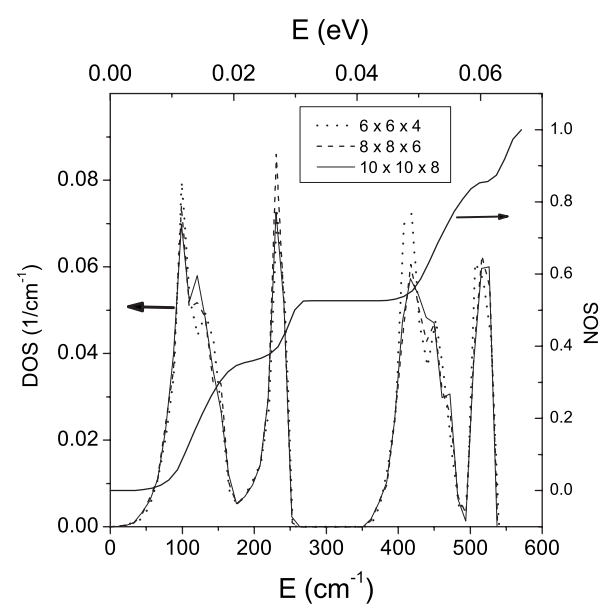

FIG. 1. The DOS calculated for the three grids of phonon wave vectors, and the number of phonon states for the $10 \times 10 \times 8$ grid normalized at maximum to unity.

the calculated phonon frequencies from the experimental ones is about $30 \mathrm{~cm}^{-1}$, so the agreement between the present calculations and the experiment is comparable to that of the previous evaluations. ${ }^{27} \mathrm{~A}$ better correspondence between the calculated and experimental phonon energies is observed for the phonon states along the $\mathrm{M}-\Gamma$ and $\Gamma-\mathrm{A}$ directions. For the $\Gamma-\mathrm{K}$ direction the calculated phonon energies are typically lower than the experimental data. However, the underestimation of the phonon energies for the states along the $\Gamma-\mathrm{K}$ direction is partly compensated by an overestimation for the states along the $\mathrm{M}-\Gamma$ direction. Besides, the phonon states nearly the $\Gamma-\mathrm{K}$ direction represent only a small portion of the states employed in the momentum averaging. So the mentioned deviations between calculated and experimental data for the $\Gamma$-K direction hardly evoke noticeable errors in the averaged indices of electron dynamics.

Figure 3 demonstrates the data necessary for the adequate choice of the broadening width $\eta$. This value should be as small as possible but providing good quality of the averaging over the $\mathbf{q}$ vectors. At the excess energy of excited electrons about 1.5 and $3.0 \mathrm{eV}$ a strong dispersion with $\eta$ and the number of $\mathbf{q}$ vectors is observed when $\eta$ is below $0.03 \mathrm{eV}$.

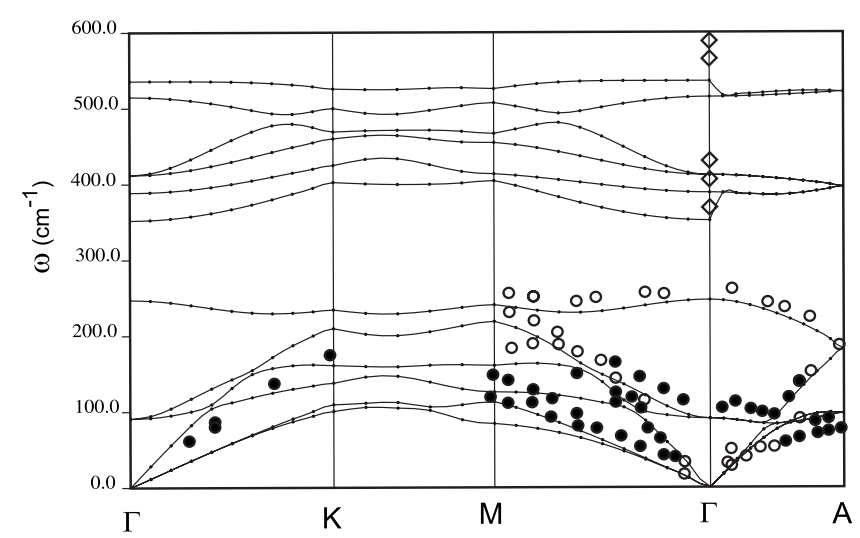

FIG. 2. The calculated phonon dispersion curves (solid lines) along symmetry directions in the Brillouin zone of the wurtzitestructure $\mathrm{ZnO}$ and the corresponding experimental data as well, open and solid circles, and diamonds (Refs. 32-34). 


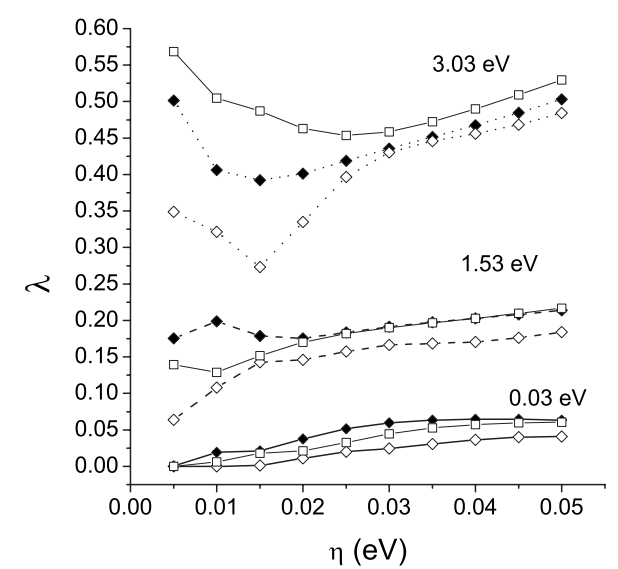

FIG. 3. The dependence of the electron-phonon coupling strength parameter $\lambda$ on the number of phonon $\mathbf{q}$ vectors in the grid and on the broadening width $\eta$ for the three excess energies of the excited electron, $0.03,1.53$, and $3.03 \mathrm{eV}$. The open diamonds are the data for the $6 \times 6 \times 4$ grid of $\mathbf{q}$, open squares are for the $8 \times 8$ $\times 6$ grid and black diamonds are for the $10 \times 10 \times 8$ grid.

Since no good convergence is obtained for such values of $\eta$, we define the value $\eta=0.03 \mathrm{eV}$ as the acceptable low limit and all the following data on the relaxation are obtained with this $\eta$ value.

In Fig. 4 we show the dependence of the electron-phonon coupling parameter $\lambda$, relaxation rate $\Gamma$ and electron energy loss at one electron-phonon scattering event $\Delta e$ on the excess energy of excited electron with different number of the phonon $\mathbf{q}$ vectors. It is evident that the $\Delta e$ value is well converged with the number of $\mathbf{q}$ vectors and is practically inde-
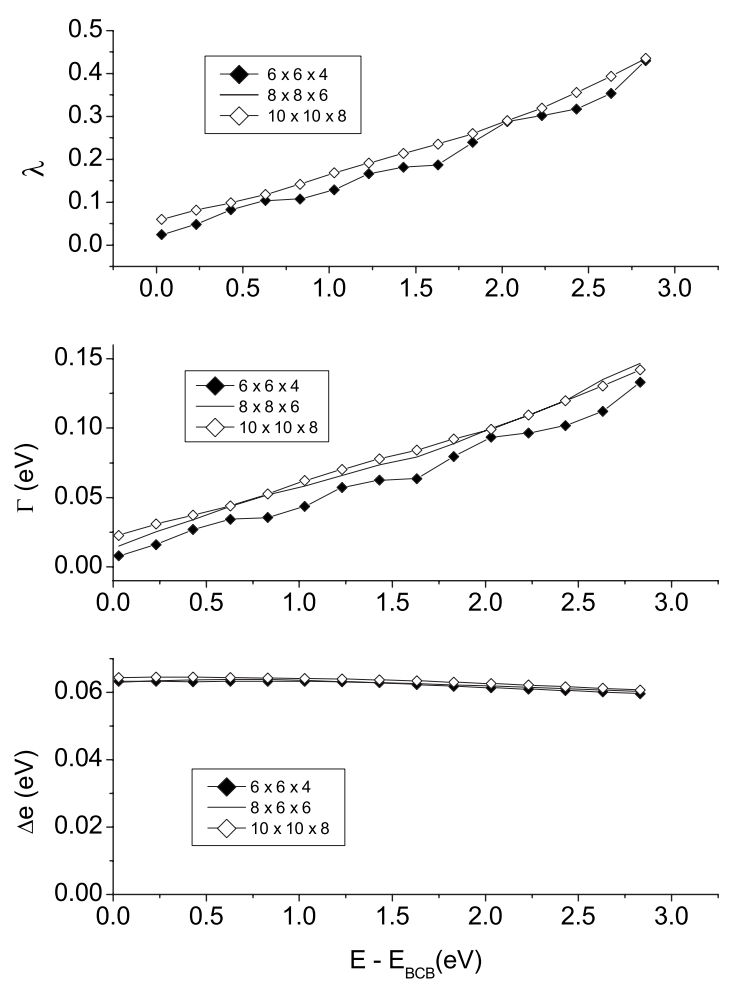

FIG. 4. The dependence of $\lambda, \Gamma$, and $\Delta e$ on the excess energy of an excited electron $E-E_{\mathrm{BCB}}$ and on the number of phonon $\mathbf{q}$ vectors in the grid.
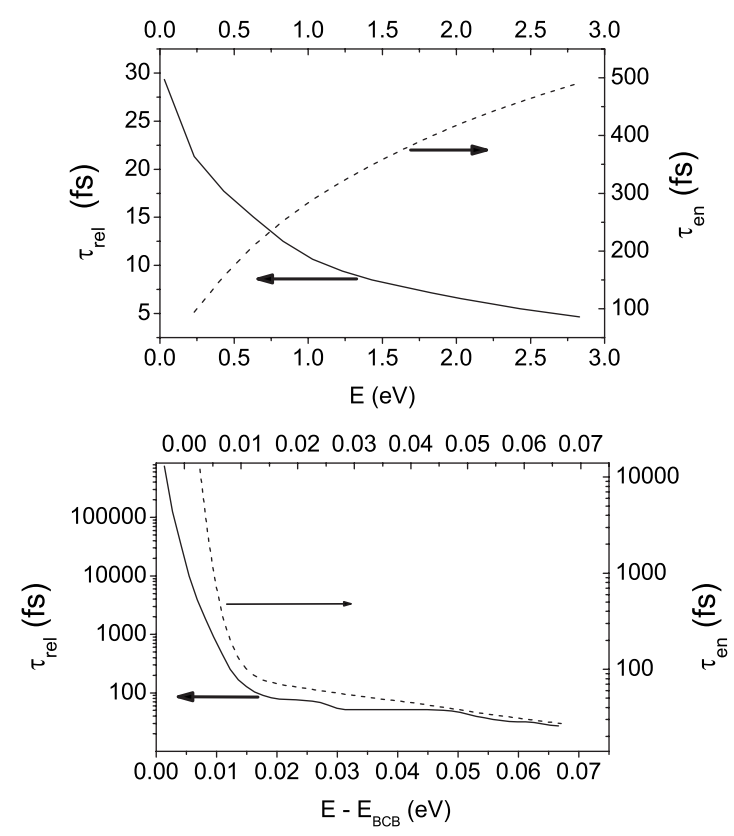

FIG. 5. The dependence of the relaxation time $\tau_{r e l}$ and energyloss time $\tau_{e n}$ on the excess energy of excited electron in the conduction band of $\mathrm{ZnO}$.

pendent on the energy of electron, hence we assume hereafter that $\Delta e=0.063 \mathrm{eV}$. As for the $\lambda$ and $\Gamma$ values, with $6 \times 6 \times 4$ grid they show irregular changes with energy and deviate from the results obtained for the denser grids. The results for the $8 \times 8 \times 6$ grid and $10 \times 10 \times 8$ grid are almost identical, that confirms a good convergence of these results with respect to the density of the grid of $\mathbf{q}$ vectors.

In Fig. 5 the dependence of the relaxation time $\tau_{r e l}$ and energy-loss time $\tau_{e n}$ on the electron excess energy is shown. Both $\tau_{r e l}$ and $\tau_{e n}$ have been evaluated for two energy intervals of interest, the first one being from zero up to the maximum phonon energy and the second being above the first one up to the value of the forbidden band gap.

The data reveal two regimes of the relaxation processes. At the electron excess energy above $\sim 0.01 \mathrm{eV}$ a fast relaxation regime is expected with both relaxation time and energy-loss time in the femtosecond time scale. At lower energy, due to smaller number of excited phonon modes, a slow picosecond relaxation is expected, with both the relaxation time and energy-loss time quickly rising with reduction in electron excess energy.

The comparison between our values of relaxation rate $\tau_{r e l}$ and available experimental data raises problems associated with the interplay between our theoretical approach and performed experiments. The characteristics studied in the timeresolved spectroscopy experiments (absorption, transmission, conductivity) depend on the electron populations $\rho(t, \epsilon)$ of the energy levels of interest. The temporal change in the population is approximated usually with the function $\rho(t, \epsilon)$ $=\rho(0, \epsilon) \exp \left[-t / \tau_{\text {exp }}(\epsilon)\right]$ where $\tau_{\text {exp }}$ is the time characterizing the rate of reduction in the energy level population. The value $\tau_{\text {exp }}$ is evaluated by adjusting to the experimental data, hence it is influenced by the effects of temperature and the effect of backflow of energy from the phonons to electrons which can be important at high fluences of excitation. The 
TABLE I. The major features of the experiments on the electron-phonon relaxation in $\mathrm{ZnO}$. Here $F$ is the fluence of excitation, $T$ is the temperature, $E$ is the energy of the studied states (with respect to the top of the valence band), ToS is the type of the studied states, $N_{\text {exc }}$ is the density of the excited electron-hole pairs, $\tau$ is the time of electron-phonon relaxation. The notations for the types of states are: SCB is for the states of the conductivity band, RSCB is for the states of the renormalized conductivity band, EHPDE is for the electronhole plasma occurring after delocalization of the exciton gas.

\begin{tabular}{|c|c|c|c|c|c|c|}
\hline Reference & $\begin{array}{c}F \\
\left(\mu \mathrm{J} / \mathrm{cm}^{2}\right)\end{array}$ & $\begin{array}{c}T \\
(\mathrm{~K})\end{array}$ & $\begin{array}{c}E \\
(\mathrm{eV})\end{array}$ & ToS & $\begin{array}{c}N_{e x c} \\
\left(1 / \mathrm{cm}^{3}\right)\end{array}$ & $\begin{array}{l}\text { Time of electron-phonon } \\
\text { relaxation (ps) }\end{array}$ \\
\hline Yamamoto et al. ${ }^{\mathrm{a}}$ & 90 & 273 & $3.21-3.27$ & RSCB & & 1 \\
\hline \multirow[t]{2}{*}{ Sun et al. ${ }^{\mathrm{b}}$} & 13 & 273 & $3.39-3.48$ & SCB & $4 \times 10^{18}$ & 0.2 \\
\hline & 100,130 & 273 & 3.33 & EHPDE & $2 \times 10^{19}, 4 \times 10^{19}$ & 0.2 \\
\hline \multirow[t]{2}{*}{ Hendry et al..$^{\mathrm{c}}$} & 200 & 30 & 3.1 & RSCB & $2 \times 10^{16}$ & 20 \\
\hline & & & 4.7 & SCB & $2 \times 10^{19}$ & 1.5 \\
\hline \multirow[t]{3}{*}{ Wen et al. ${ }^{\mathrm{d}}$} & 1000 & 273 & 3.54 & SCB & $1 \times 10^{20}$ & $<1$ \\
\hline & & & 3.34 & EHPDE & & 7 \\
\hline & & & 3.26 & RSCB & & 34 \\
\hline
\end{tabular}

\footnotetext{
${ }^{\mathrm{a}}$ Reference 12.

${ }^{\mathrm{b}}$ Reference 13 .

${ }^{\mathrm{c}}$ Reference 9.

${ }^{\mathrm{d}}$ Reference 6.
}

backflow can lift the electrons from the lower energy levels to the level of interest, thus increasing the relaxation time. An essential role of such process has been remarked in the pump-probe experimental studies of Sun et al. ${ }^{13}$ In our approach the temperature factor in neglected and our relaxation times refer to the relaxation of a single particle, which is not the case of experimental works. So our relaxation time is not identical to the experimental relaxation time $\tau_{\text {exp }}(\epsilon)$.

In Table I we have collected the major information on the experiments employed for the studies of the electron-phonon relaxation of the excited states in $\mathrm{ZnO}$. The experiments of Yamamoto et al. ${ }^{12}$ have been performed for $230 \mathrm{~nm}$ thick epitaxial films of $\mathrm{ZnO}$, the experiments of Sun et al. ${ }^{13}$ have been done for $80 \mathrm{~nm}$ thick nanorods, and the results of Hendry et al. ${ }^{9}$ were obtained from measurements of bulk $\mathrm{ZnO}$ samples. So the data obtained in these experiments can be associated with the bulk $\mathrm{ZnO}$. On the contrary, the results of Wen et al. ${ }^{6}$ were derived from measurements of $\mathrm{ZnO} /$ $\mathrm{ZnMgO}$ multiple quantum wells with thin $(2$ and $5.5 \mathrm{~nm})$ layers of $\mathrm{ZnO}$ and $\mathrm{ZnMgO}$. Nevertheless, we will show later that the results of Wen et al. are in reasonable agreement with the others experiments.

In Refs. 6, 12, and 13 the study of the excited electron dynamics in $\mathrm{ZnO}$ was performed by means of the pumpprobe techniques with the energy of the pump and probe quanta in optical range. The experiments were carried out at room temperature and with different fluences of excitation energy. In the Ref. 12 the cooling of electron-hole plasma in $\mathrm{ZnO}$ was studied with high density of excitation, up to $280 \mu \mathrm{J} / \mathrm{cm}^{2}$. Yamamoto et al. ${ }^{12}$ evaluated the time of cooling of electron-hole plasma as being about 1 ps. With high density of excitation the backflow raises the relaxation time, so the cooling time of Yamamoto et al. is expected to be longer than our $\tau_{r e l}$ values, which really takes place. A paradoxical feature of the Yamamoto et al. results is that the relaxation time has been determined from the absorption spectrum at the energy $\geq 3.20 \mathrm{eV}$, lower than the forbidden bang gap, $3.37 \mathrm{eV}$. The authors explained the possibility of such absorption by the renormalization of the band gap in the presence of high number of carriers excited by the pump pulse. The bottom energy of the renormalized conduction band is difficult to derive from the data of Yamamoto et al.; it is, however, clear that this energy is less than $3.2 \mathrm{eV}$. Hence it would be incorrect to compare the data of Yamamoto et al. with our data at a definite electron energy.

More satisfactory is the comparison of our $\tau_{r e l}$ values with the data of Sun et al. ${ }^{13}$ They studied the electron dynamics at two excitation energies, $3.39-3.48 \mathrm{eV}$ and $3.33 \mathrm{eV}$. In the experiments at $3.39-3.48 \mathrm{eV}$ the electron relaxation time was evaluated with the pump energy fluence significantly less intensive than in the work of Yamamoto et al. So one may expect that the renormalization of the band gap in Ref. 13 was smaller than in the Yamamoto's experiments and the process of electron relaxation is more similar to the relaxation of a single carrier. At the excitation energy $\sim 0.04 \mathrm{eV}$ above the nonrenormalized band gap width Sun et al. have obtained $\tau_{\text {exp }}=200$ fs. The validity of their evaluations for $\tau_{\text {exp }}$ was supported by the experiments at the energy of 3.33 $\mathrm{eV}$. This energy, below the forbidden band gap, corresponds to the excitonic states, so at low fluence of excitations the authors refer the experimental relaxation time for such energy to the exciton recombination. At the excitation fluence about $130 \mu \mathrm{J} / \mathrm{cm}^{2}$ they find a second process of relaxation with the time constant $\sim 200$ fs. Since this occurs at the concentration of electron-hole pairs higher than the Mott's one, $3.7 \times 10^{19} 1 / \mathrm{cm}^{3},{ }^{13}$ they refer this time constant to the cooling, due to the electron-phonon coupling, of the electron-hole plasma which appears as a result of the interactions between excitons.

We have at $E-E_{\mathrm{BCB}}=0.04 \mathrm{eV} \tau_{\text {rel }}=46 \mathrm{fs}$, still shorter by a factor of 4 than the Sun's value of 200 fs. A presumable origin of this deviation is a still high intensity of excitations 
in experiments, due to which the backflow of energy to excited electrons takes place raising the relaxation time. Notice that much lower energy fluence is employed in the pumpprobe experiments on the single electron relaxation in metals. ${ }^{35}$ In such experiments roughly about $10^{6}$ electrons $/ \mathrm{cm}^{3}$ are excited, significantly less than $10^{18}$ electrons $/ \mathrm{cm}^{3}$ in the work of Sun et al.

The validity of our evaluations of $\tau_{\text {rel }}$ can also be checked by comparison with the experimental data of Hendry et al. ${ }^{9}$ The authors studied the electron dynamics at two energies of pump quanta, 4.7 and $3.1 \mathrm{eV}$, and with high fluence of excitation, $200 \mu \mathrm{J} / \mathrm{cm}^{2}$. The relaxation time was derived from the temporal evolution of the transient conductivity as a reaction of the system to the applied probe quanta of tetrahertz energy. In the first case the plasma of electron-hole pairs was generated with the density of $2 \times 10^{19} 1 / \mathrm{cm}^{3}$. The authors derived the time of cooling about $1.5 \mathrm{ps}$, which is comparable with the data of Jamamoto et al., 1 ps, Sun et al., 200 fs. Moreover, the higher value of $\tau_{\exp }$ of Hendry et al. is compatible with the fluence of excitation in their experiments higher than in the works of Yamamoto et al. and Sun et al.

In the second case the number of electron-hole pairs was about $10^{16} 1 / \mathrm{cm}^{3}$, less than in the work of Sun et al. ${ }^{13}$ Since the excitation energy, $3.1 \mathrm{eV}$, is less than the forbidden band gap and the fluence of excitation is high, the relaxation process should be referred to the cooling of the states of the renormalized conduction band. Unexpectedly, in this case Hendry et al. evaluated the time of cooling as $20 \mathrm{ps,} \mathrm{much}$ higher than the time of cooling with low fluence of excitation from the works of Yamamoto et al., ${ }^{12}$ Sun et al. ${ }^{13}$ One can rationalize this result if one approves that the states studied at the excitation energy of $3.1 \mathrm{eV}$ are very close to the bottom of the renormalized conductivity band. Since the energy of the probe quanta in Hendry's experiments was about $1 \mathrm{THz}=0.004 \mathrm{eV}$, we can compare the data of Hendry et al. with our data at such excess energy, 46 ps. The difference between experimental and theoretical data is quite sensible, taking into account the simplifications of our calculations at low energy, first of all omitting the calculations of electronphonon matrix elements.

The comparison with the data of Wen et al. ${ }^{6}$ confirms the validity of the previous speculations. The experiments were carried out at the fluence of excitation $\sim 1 \mathrm{~mJ} / \mathrm{cm}^{2}$; the energies of the studied states were from 3.26 to $3.54 \mathrm{eV}$. With such fluence of excitation, the excitation near $3.26 \mathrm{eV}$ should be to the states of the renormalized conduction band. When the energy of excitation is close to the middle of this energy interval, the excited states are the states of electron-hole plasma after the Mott's transition in the gas of excitons. At the top of this energy interval one should observe the excitation to the normal conductivity band states. So, basing on the previous discussion, one should observe with the decrease in the excitation energy an essential increase in the relaxation time. This is just the case of the data of Wen et al., see Table I and more complete data in Ref. 6.

One more indirect confirmation of the fast electronphonon relaxation of the electrons in the conduction band of $\mathrm{ZnO}$ is contained in the Ref. 36. In this work the rate of electron-phonon relaxation has been evaluated for the highly $n$-doped $\mathrm{ZnO}$ with the concentration of carriers in the conduction band equal to about $10^{20} 1 / \mathrm{cm}^{3}$. The pumping was in the IR region with the energy of quanta of $0.709 \mathrm{eV}$, and the probing of transient transmission was in the UV region with the energy of quanta from 3.18 to $3.40 \mathrm{eV}$. So probed was the relaxation of the electrons in the conduction band with the excess energy up to about $0.7 \mathrm{eV}$; the authors evaluated the time of relaxation as about $500 \mathrm{fs}$.

\section{CONCLUSIONS}

Basing on the density-functional perturbation theory, we performed the first-principle evaluations for the electronphonon coupling parameters, for the relaxation time and time of energy loss of excited electrons in the conduction band of zinc oxide. The calculations have been examined with respect to the choice of the number of basic plane waves, the number of wave vectors in the Monkhorst-Pack grid and the broadening width for averaging near the energy of excited electron. The calculations demonstrate two types of excited electron dynamics. For electronic excited states with excess energy higher than the maximum phonon energy the electron dynamics occurs in the femtosecond region. In this region the relaxation time and energy-loss time have different trends with increase in energy, the reduction in the first one and the increase in the second. For the excited states with excess energy less than $0.01 \mathrm{eV}$ the picosecond dynamics is predicted. In this case both the relaxation time and the energyloss time sharply raise with the decrease in the energy of electrons, which is associated with the reduction in the phonon phase space. Notice that these results are in some aspects analogous to the results of the papers ${ }^{37,38}$ where the phononmediated excited electron relaxation on metal surfaces has been studied. The authors also found two regimes of relaxation, the slow one at the excitation energy less than $\sim 30 \mathrm{meV}$ and the fast one at higher energies.

The comparison of the calculated relaxation time with the experimental data shows sensible agreement, thus confirming existence of the two types of electron dynamics. As for the calculated energy-loss time, we are not aware about any experimental data that can be compared with our prognosis. However, a qualitative conclusion useful for better understanding of the photochemical reactions on the surface of zinc oxide can be drawn. In real experiments on photocatalyses the electrons can be excited by UV emission much above the BCB. However, since the energy-loss time for the energy levels less than $0.01 \mathrm{eV}$ far from the $\mathrm{BCB}$ are three orders higher then those for higher energy, one should expect that because of the fast femtosecond relaxation from the higher states the photocatalytic properties are determined almost exclusively by the states at the bottom of the conduction band.

\section{ACKNOWLEDGMENTS}

We acknowledge partial support from the University of the Basque Country (Grant No. GIC07IT36607), the Spanish Ministerio de Ciencia y Tecnologia (Grant No. FIS200766711-C02-01) and the Ikerbasque Fellowship foundation. 
${ }^{1}$ M. Joseph, H. Tabata, and T. Kawai, Jpn. J. Appl. Phys., Part 2 38, L1205 (1999).

${ }^{2}$ M. Venkatesan, C. B. Fitzgerald, J. G. Lunney, and J. M. D. Coey, Phys. Rev. Lett. 93, 177206 (2004).

${ }^{3}$ H. Lu, H. Li, L. Liao, Y. Tian, M. Shuai, J. C. Li, M. F. Hu, Q. Fu, and B. P. Zhu, Nanotechnology 19, 045605 (2008).

${ }^{4}$ Y. Ni, X. Cao, G. Wu, G. Hu, Z. Yang, and X. Wei, Nanotechnology 18, 155603 (2007).

${ }^{5}$ O. I. Girdasova, V. N. Krasilnikov, L. Iu. Buldakova, M. Iu. Yanchenko, and O. V. Koriakova, Izv. Ross. Akad. Nauk, Ser. Fiz. 73, 1176 (2009)

${ }^{6}$ X. M. Wen, J. A. Davis, D. McDonald, L. V. Dao, P. Hannaford, V. A. Coleman, H. H. Tan, C. Jagadish, K. Koike, S. Sasa, M. Inoue, and M. Yano, Nanotechnology 18, 315403 (2007).

${ }^{7}$ J. Takeda, N. Arai, Y. Toshine, H.-J. Ko, and T. Yao, Jpn. J. Appl. Phys., Part 1 45, 6961 (2006).

${ }^{8}$ F. Bertram, J. Christen, A. Dadgar, and A. Krost, Appl. Phys. Lett. 90, 041917 (2007).

${ }^{9}$ E. Hendry, M. Koeberg, and M. Bonn, Phys. Rev. B 76, 045214 (2007).

${ }^{10}$ T. Makino, N. T. Tuan, H. D. Sun, C. H. Chia, Y. Segawa, M. Kawasaki, A. Ohtomo, K. Tamura, T. Suemoto, H. Akiyama, M. Baba, S. Saito, T. Tomita, and H. Koinuma, Appl. Phys. Lett. 78, 1979 (2001).

${ }^{11}$ T. Makino, Y. Segawa, M. Kawasaki, and H. Koinuma, Semicond. Sci. Technol. 20, S78 (2005).

${ }^{12}$ A. Yamamoto, T. Kido, T. Goto, Y. Chen, T. Yao, and A. Kasuya, Appl. Phys. Lett. 75, 469 (1999).

${ }^{13}$ C. K. Sun, S. Z. Sun, K. H. Lin, K. Y. J. Zhang, H. L. Liu, S. C. Liu, and J. J. Wu, Appl. Phys. Lett. 87, 023106 (2005).

${ }^{14}$ V. P. Zhukov, F. Aryasetiawan, E. V. Chulkov, I. G. de Gurtubay, and P. M. Echenique, Phys. Rev. B 64, 195122 (2001).

${ }^{15}$ V. P. Zhukov, F. Aryasetiawan, E. V. Chulkov, and P. M. Echenique, Phys. Rev. B 65, 115116 (2002).

${ }^{16}$ I. Campillo, V. M. Silkin, J. M. Pitarke, E. V. Chulkov, A. Rubio, and P. M. Echenique, Phys. Rev. B 61, 13484 (2000).

${ }^{17} \mathrm{G}$. Grimvall, The Electron-Phonon Interactions in Metals (North-Holland, Amsterdam, 1981).

${ }^{18} \mathrm{Ph}$. Hofmann, I. Yu. Sklyadneva, E. D. L. Rienks, and E. V.
Chulkov, New J. Phys. 11, 125005 (2009).

${ }^{19}$ E. N. Zein, Fiz. Tverd. Tela (Leningrad) 26, 3024 (1984) [Sov. Phys. Solid State 26, 1825 (1984)].

${ }^{20}$ S. Baroni, S. de Gironcoli, and A. Dal Corso, Rev. Mod. Phys. 73, 515 (2001).

${ }^{21}$ J. Sjakste, V. Tyuterev, and N. Vast, Phys. Rev. B 74, 235216 (2006).

${ }^{22}$ J. Sjakste, N. Vast, and V. Tyuterev, Phys. Rev. Lett. 99, 236405 (2007).

${ }^{23}$ J. Y. Zhang, L. J. Zhang, T. Cui, Y. L. Niu, Y. M. Ma, Z. He, and G. T. Zou, J. Phys.: Condens. Matter 19, 425218 (2007).

${ }^{24}$ V. P. Zhukov, E. V. Chulkov, Phys. Usp. 52, 105 (2009).

${ }^{25} \mathrm{http}$ ://www.quantum-espresso.org, http://www.pwscf.org

${ }^{26}$ R. Sikora, J. Phys. Chem. Solids 66, 1069 (2005).

${ }^{27}$ J. Serrano, A. H. Romero, F. J. Manjo'n, R. Lauck, M. Cardona, and A. Rubio, Phys. Rev. B 69, 094306 (2004).

${ }^{28}$ M. Usuda, N. Hamada, T. Kotani, and M. van Schilfgaarde, Phys. Rev. B 66, 125101 (2002).

${ }^{29}$ F. Aryasetiawan and O. Gunnarsson, Rep. Prog. Phys. 61, 237 (1998); W. G. Aulbur, L. Jönsson, and J. W. Wilkins, Solid State Phys. 54, 1 (2000).

${ }^{30}$ S. Massidda, R. Resta, M. Posternak, and A. Baldereschi, Phys. Rev. B 52, R16977 (1995).

${ }^{31}$ J. Bardeen and W. Shockley, Phys. Rev. 80, 72 (1950).

${ }^{32}$ A. W. Hewat, Solid State Commun. 8, 187 (1970).

${ }^{33}$ K. Thoma, B. Dorner, G. Duesing, and W. Wegener, Solid State Commun. 15, 1111 (1974).

${ }^{34}$ J. Serrano, F. Widulle, A. H. Romero, A. Rubio, R. Lauck, and M. Cardona, Phys. Status Solidi B 235, 260 (2003).

${ }^{35}$ M. Bauer and M. Aeschlimann, J. Electron Spectrosc. Relat. Phenom. 124, 225 (2002).

${ }^{36}$ H. Ye, P. M. Fauchet, S. Mathukumar, and Y. Lu, Proc. SPIE 4280, 114 (2001).

${ }^{37}$ A. Eiguren, B. Hellsing, F. Reinert, G. Nicolay, E. V. Chulkov, V. M. Silkin, S. Hüfner, and P. M. Echenique, Phys. Rev. Lett. 88, 066805 (2002).

${ }^{38}$ A. Eiguren, B. Hellsing, E. V. Chulkov, and P. M. Echenique, Phys. Rev. B 67, 235423 (2003). 